\title{
Perspectives in psoriasis, psoriatic arthritis, non-alcoholic fatty liver disease and atherosclerosis in psoriasis
}

\author{
Iqbal Bukhari', Mona Ismail ${ }^{2}$, Manal Hasan ${ }^{2}$, Abdulaziz Alzahrani ${ }^{3}$ \\ ${ }^{1}$ Dermatology Department, Imam Abdulrahman Bin Faisal University and King Fahd Hospital of the University, Dammam, Saudi \\ Arabia, ${ }^{2}$ Internal Medicine department, Imam Abdulrahman Bin Faisal University and King Fahd Hospital of the University, Dammam, \\ Saudi Arabia, ${ }^{3}$ Departments of Dermatology, Venereology, Allergology and Immunology, Dessau Medical Center, Dessau, Germany \\ Corresponding author: Prof. Iqbal Bukhari, E-mail: ibukhari@iau.edu.sa
}

\begin{abstract}
Psoriasis is a disease of chronic systemic inflammation that involves not only the skin, but also internal organs. The frequency of Non-alcoholic fatty liver disease was found to be significantly greater in psoriasis patients with increased risk of atherosclerosis and cardiovascular disease. A large number of immunes is found in Psoriatic skin and this immune produce chemokine's, cytokine and inflammatory molecules. The exact role of genetics in psoriasis is still unclear and an overlap between some psoriasis loci and those identified in other autoimmune or inflammatory diseases has been reported.
\end{abstract}

Key words: Psoriasis; Genetics; Arthritis; Fatty liver; Atherosclerosis; Obesity

\section{INTRODUCTION}

Psoriasis affects 2-3\% of the European population. It is common found less in an individual of Asian descent $(0.1 \%$ or less $)$ and is exceedingly rare in Africa [1]. Large number of immunes is found in Psoriatic skin and this immune produce chemokine's, cytokine and inflammatory molecules. The genetics basis of psoriasis has been unclear yet, whether they reflect defects of the immune system or of the skin. It has been reveal by Genome-Wide association that genetic susceptibility factors which play a role in the formation of immune cell found in psoriasis or in the proliferation epidermal cell and skin barrier formation. Furthermore, as many as $10-30 \%$ of patient with psoriasis develop an inflammatory arthritis which causes the destruction of joints if it is not properly treated in aggressive manner. It is now universally acknowledged that psoriasis and Psoriatic arthritis are consistent with a multifactorial pattern of inheritance.

\section{Pre-Genome Wide Association Studies in Psoriasis Genetics}

The earliest genetic studies revealed association with human leukocyte antigen (HLA) class 1 alleles, and the strongest association was with the HLA-C allele. Approximately 10 genome-wide linkage scans, primarily with polymorphic microsatellites, led to the identification of over 20 possible linked regions. Some of which are: PSORS1 on 6p21.3 [2], PSORS2 on 17q [3], PSORS3 on 4q [4], PSORS4 on 1cenq21 [5], PSORS5 on 3q21 [6], PSORS6 on 19p [7], PSORS7 on $1 \mathrm{p}$ [8], and PSORS9 on 4q31 [9].

\section{Psoriasis genetic associations}

\section{Inflammatory genes}

GWAS studies have been performed primarily in populations of European and Asian till date, and the most highly significant associations that are found in both population are with SNPs from the MHC class I region that encodes the HLA molecules HLAA, HLAB and HLAC. The psoriasis-associated with SNPs are nearly to the gene encoding HLAC [10-12] and this have form several risk Gene that are HLAC [10-12], IL12B [13], IL23A, IL23R, TNFAIP3, TNIP1 [11], IL2\IL2 1 [10], SLC12A8 [14], ZNF313 [5], HBD [15] and LCE [12]. Additional two independent MHC locihave also confer the risk of psoriasis in both European and Chinese populations. One of the Mhc

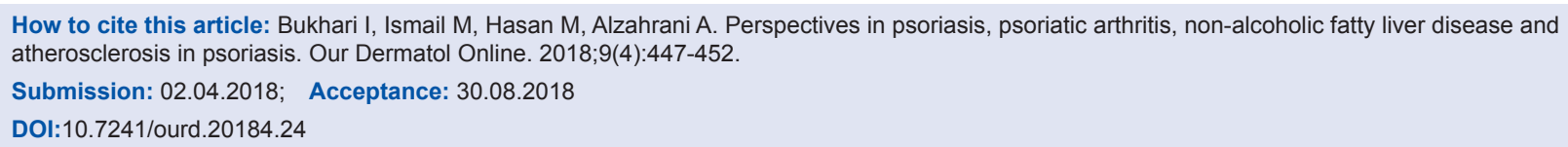


loci is found within the chromosome 6 open reading frame 10 (c6orfl0), and the second locus is $30 \mathrm{~kb}$ in size centromeric of HLAB and $16 \mathrm{~kb}$ telomeric of the MHC class I polypeptide related sequence A gene, MICA [16].

\section{Barrier development genes}

There is a new evidence that skin barrier functions may also plays a role in susceptibility to develop psoriasis. The Epidermal Differentiation Complex lies on human chromosome lq21, spans $2 \mathrm{Mb}$ and encodes at least 45 genes that play a role in the generation or maintenance of the epidermis. Many genes of the Epidermal Differentiation Complex are upregulated in psoriatic lesions suggesting the underlying alterations in coordinate regulation of genes of this complex $[17,18]$.

\section{Psoriasis risk factors shared by other autoimmune or inflammatory diseases}

To date only one genome-wide scan has been completed in psoriatic arthritis (PsA), and this study localized a candidate region on chromosome 16q. Association of psoriasis and PsA with alleles in the MHC region has been recognized for over three decades, and presently there are plethora of association studies for both of these disorders with HLA alleles. Overlap between some psoriasis loci and those identified in other autoimmune or inflammatory diseases has been reported. The same variant of IL23R is associated with Crohn's disease, PsA, and ankylosing spondylitis $[10,19,20]$. This is consistent with the role of IL23R in Th17 cell activation and with the fact that these cells have a pathogenic role in several other inflammatory diseases including Crohn's disease and multiple sclerosis [21].

\section{Genetics of Psoriatic Arthritis}

Approximately $25 \%$ of patients also develop psoriatic arthritis (PsA), a common, debilitating auto-immune disease belonging to the family of spondyloarthritides [22] . HLA alleles have been associated with both psoriasis and PsA [23]. were described as being [24]. TNF- $\alpha-238$ polymorphism and PsA [25] on psoriasis subjects of European ethnicity [10].

\section{Genetics of Psoriatic Arthritis}

Approximately $25 \%$ of patients also develop psoriatic arthritis (PsA), a common, debilitating auto-immune disease belonging to the family of spondyloarthritides [22]. Different number of studies base on association on a candidate-gene approach has been conducted to identify genes underlying susceptibility to PsA. Since the PSORS1 locus within the $\mathrm{MHC}$ region on 6p provides the strongest linkages with psoriasis in Genome wide linkage scan, also candidate gene within this region have been investigated. The number of genes for a gene dense region code are important in the immune response, including HLA and non-HLA genes. HLA alleles have been associated with both psoriasis and PsA [23]. It is not clear whether the associate HLA described are with psoriasis or with PsA, or both because most of the patient with PsA have psoriasis. HLA alleles that are peculiar to PsA are HLA-B27, B7, B38, and B39.HLA-B13, -B16, and its splits -B38 and $-\mathrm{B} 39, \mathrm{~B} 17$ and $\mathrm{Cw} 6$ were described as being psoriasis associated arthristis or not [24]. The possible biologic significance that are assciated with psoriasis is recognize to be nine genes and this genes include HLA-B, HLA-C, PSORS1C3, OTF3, HCR, SPR1, SEEK1, corneodesmosin (CDSN), and TNF- $\alpha$ [25-31]. Genomic DNA sequences and recombinant haplotypes suggested that HLA-Cw* 0602 is the allele diseases at PSOR1. It has been noted that gene within this region have been investigated with PsA [32]. A study from a metaphysis shows that there is an association between TNF- $\alpha-238$ polymorphism and PsA [33]. A recent fine mapping of gene in MHC region has been observe the association of PsA with SNP rs1 1507 [34-35]. Therefore, susceptibility locus for PsA may lie more centromeric to that of psoriasis and these is closer to HLA-B. PsA associated studies has recognized that a numbers of genes outside the chromosome 6p are IL-23R, IL-1, and killer-cell immunoglobulin like receptor genes $[33,35,36]$. The study of GWAS on PsA has not been formally shown. However GWAS on psoriasis subjects of European ethnicity, there were three loci that is associated with PsA when compared to normal controls (HLA-C, IL-12B, and TNIP1). it has been shown that between PsA and psoriasis alone, there is a statistical significant differences at three loci (HLA-C, IL-12B and IL-23R). The loci that more strongly associated with psoriasis alone are HLA-C and IL-23R, and IL-12B with PsA [11]. Another GWAS identified a novel PsA locus on chromosome 4q27 that harbors the interleukin 2 (IL-2) and interleukin 21 (IL21) genes [10].

\section{Psoriasis, Non-alcoholic Fatty Liver Disease and Obesity}

Obesity is a significant and growing problem worldwide and it has also been linked to the onset of psoriasis. In the Nurses' Health Study II, increased body mass index (BMI) correlated with an increased incident rate of psoriasis, and hip circumference and waist- 
to-hip ratio were all associated with a higher risk of incident psoriasis [37]. The psoriasis that are associated with metabolic syndrome and increase risk of cardiovascular disease are non-alcoholic fatty liver disease (NAFLD) and chronic plague. NAFLD is the hepatic manifestation of metabolic syndrome, with its key component being visceral obesity [38]. The prevalence of NAFLD is $10-24 \%$ of the general population worldwide, increasing to $57.5-74 \%$ in obese individuals. The mortality was increased in patients with NAFLD compared with the general population and in the National Health and Nutrition Examination Survey (NHANES III) study, the NAFLD cohort had both increased overall mortality and liver-related mortality compared with individuals without liver disease [39]. While in most patients, NAFLD does not progress beyond simple steatosis, it may progress to Non-Alcoholic Steato-Hepatitis (NASH). The prevalence of NASH also correlates with obesity, with waist-to-hip ratio and abdominal obesity reported to be predictors of NASH. Diagnosing patients with NAFLD and identifying those with NASH is challenging, as they are generally asymptomatic. Clinical presentation and current radiological modalities may not be reliably to diagnose NASH, while non-invasive biomarkers remain to be fully established. The frequency of NAFLD was found to be significantly greater in psoriasis patients (47\%) vs. matched controls (28\%). Indeed, the relationship strengthened with increasing psoriasis severity [40]. Collectively, these observations support the early recognition of NAFLD in psoriasis patients, the results of which may dictate treatment regimens to avoid potentially liver toxic therapies, such as methotrexate. Positive correlations have been reported among cumulative methotrexate dose, risk factors (e.g. obesity) and progression of NAFLD. Gram for gram, psoriasis patients are also twice as likely to develop hepatic complications from methotrexate as a patients with rheumatoid arthritis (RA). The prevalence of obesity and being overweight are increasing in Saudi Arabia reaching 35.5\% [41]. Thus, reduction of weight are of considerable importance to public health [42].

\section{Psoriasis Inflammation and Endothelial Dysfunction}

Psoriasis inflammation may act independently in promoting an accelerated atherosclerosis by eliciting endothelial dysfunction and oxidative stress similarly to other chronic inflammatory systemic diseases, such as rheumatoid arthritis, systemic lupus erythematosus, and Crohn's disease [43]. Psoriasis inflammation is characterized by high levels of TNF-a, IFN-a, IFN-g, IL-1, IL-6, and IL-17, which are released by keratinocytes and inflammatory cells infiltrating skin and joint tissues [44]. These cytokines could also boost several proatherogenic functions of the liver, adipose tissue, and skeletal muscle, including liver production of C-reactive protein (CRP), dyslipidemia, production of proinflammatory adipokines, and insulin resistance, generating lipid abnormalities and culminate in the development of NAFLD and resulting in endothelial dysfunction. Cytokines can also mediate several metabolic effects that can in short term result to inappropriate response to injury or infection, but on a chronic basis, its prove detrimental by accelerating the development of atherosclerosis and predisposing to thrombosis. Endothelial dysfunction is the critical early step in the process of atherogenesis, and it is commonly investigated by measuring arterial stiffness. Arterial stiffness has been found increased in psoriasis patients independently of the other cardiovascular risk factors $[45,46]$.

\section{NAFLD and Cardiovascular Disease}

Giving the fact that NAFLD is usually an asymptomatic disorder, it is often unrecognized in everyday clinical practice. Therefore, patient with NAFLD have no symptoms, and aminotransferase levels which are used as a marker of liver damage, are within normal values in almost half of all patients. Type 2 diabetes (T2DM) is strongly associated with NAFLD and has been linked to increased cardiovascular disease (CVD) risk. It is characterized by insulin resistance and mitochondrial dysfunction ${ }^{6}$. Indeed, there is a gradual increase in the severity of insulin resistance in the range of NAFLD which may contribute to the evolution of liver damage. Also, it is associated with an increased risk of kidney disease in subjects with multiple CVD risk factors and tends to be considered as an independent CVD marker [47]. Diabetes, dyslipidemia, hypertension and CVD coexist more frequently in individuals with NAFLD [48]. Hepatic steatosis has been linked to visceral adiposity, low serum HDL, high serum triglycerides, and proinflammatory biomarkers such as CRP and has been shown to be associated with an increased risk of cardiovascular events independent of these other variables in diabetic patients [49].

\section{PNPLA3 Gene polymorphism and Carotid Atherosclerosis}

Identifying the underlying genetic factors for any disease locus relies intimately on data collected through 
genome-wide association (GWA) initiatives. These studies survey genotypic-phenotypic associations among large population based cohorts [50]. Recent GWAs have isolated a number of single nucleotide polymorphisms (SNPs) linked to either increased hepatic fat content or elevated liver enzymes or coronary heart disease [50-52]. The patatin-like phospholipase domain-containing protein 3 (PNPLA3) gene locus on chromosome 22 indicate one of the most investigated polymorphism in fatty liver diseases. Heterozygote carriage of the I148M minor allele in particular is associated with more hepatic triglyceride levels in 2 mixed population studies conducted in North America and Europe $[51,53,54]$. PNPLA3-148M homozygotes showed an even greater propensity for hepatic fat accumulation; triglyceride levels were two-fold higher in this group compared to non-carriers [1]. The variant allele surfaced most frequently in Hispanic persons, followed by those of European descent and least often in African Americans. On the contrary, a minor variant referred to as PNPLA3-S4531 was commonly detected in African Americans and furthermore associated with a lower hepatic fat burden in these individuals. The exact mechanism by which the PNPLA3-148M variant exerts its effects remains largely unknown. In humans PNPLA3 expresses most abundantly in the liver and to a larger extent in obese individuals [55]. Evidence from in vitro experiments suggests that PNPLA3 displays both lipolytic and lipogenic activity [56,57]. The PNPLA3-148M variant may promote fat accumulation by limiting triglyceride hydrolysis [55]. However, to what degree PNPLA3 participates in triglyceride hydrolysis remains controversial [55,57-59]. Targeted PNPLA3 deletion for example did not influence triglyceride hydrolysis or metabolic functions in animal models [57]. A marked up-regulation of PNPLA3 in response to feeding has led investigators to alternatively propose a function for PNPLA3 in lipid remodelling rather than catabolism [60]. Further functional studies should help assess the precise physiological role of PNPLA3 in hepatic lipid metabolism. Despite the strong association between fatty liver and both insulin resistance and glucose intolerance both studies which evaluated a Southern European population failed to find an independent association between PNPLA3$148 \mathrm{M}$ and features of metabolic syndrome including fasting serum insulin, HOMA-IR, triglycerides, total cholesterol, or HDL-cholesterol [14,17]. The Dallas Heart Study reported a similar finding [51].

Different lines of evidence, including cross sectional and prospective studies, showed that NAFLD patients are at high risk of cardiovascular dysfunction/events, identifying conventional cardiometabolic alterations and the extremity of liver damage as risk factors $[61,62]$.

\section{GCKR Functional Gene Variants and Atherosclerosis}

Glukokinase (GCK) is the most overriding glucose enzyme (phosphorylating enzymes) that is present in the liver and Pancreatic islets, is also known as islets of Langerhans, which have small clusters of cells scattered throughout the pancreas. Pancreatic islets contain several types of cells, including beta cells that produce the hormone insulin which act as physiological glucosesensor. This regulatory protein (Glukokinase regulatory protein) present in pancreatic islets and the liver form an inactive heterodimer. The GCKR that is $27 \mathrm{~kb}$ is located on chromosome 2p23 that contain encodes a $68 \mathrm{kDA}$ protein 19 exons. The association study of wide genome has showed that the common functional variants of the GCKR gene are associated with insulin levels, fasting plasma glucose, and both serum triglycerides and low/ high-density lipoprotein cholesterol levels, thus, single nucleotide polymorphisms [63-65]. The variant that is common in GCKR gene has been reported to be in association with increase in more CRP levels, which indicates a good atherosclerotic marker [66].

\section{IN CONCLUSION}

Psoriasis is a disease of chronic systemic inflammation, it therefore recommended to reduce the risk of associated comorbidities such as psoriatic arthritis, NAFLD and CVD by early recognition and diagnosis. Chronic inflammation, mediated by either proinflammatory adipokines or skinderived cytokines, may contribute to fatty liver disease development by increasing insulin resistance which in turn promotes hepatic lipid accumulation in patients with psoriasis and CVD risk. Further studies are needed to better understand the role of genetics and inflammatory markers. It is also essential to screen for comorbidities and hepatic ones in patients with psoriasis.

\section{ABBREVIATIONS}

HLA: Human leukocyte antigen GWA: Genome-wide association

SNP: Single nucleotide polymorphisms

MHC: Major histocompatibility complex

PS: Psoriasis

PsA: psoriatic arthritis 
www.odermatol.com

CDSN: Corneodesmosin

IL: Interleukin

BMI: Body mass index

NAFLD: Non-alcoholic fatty liver disease

NHANES: Nutrition Examination Survey

NASH: Non-alcoholic steatohepatitis

CRP: C-reactive protein

T2DM: Type 2 diabetes mellitus

CVD: Cardiovascular disease

HDL: High density lipoproteins

PNPLA3: Patatin-like phospholipase domaincontaining protein 3

GCK: Glucokinase

\section{Statement of Human and Animal Rights}

All procedures followed were in accordance with the ethical standards of the responsible committee on human experimentation (institutional and national) and with the Helsinki Declaration of 1975, as revised in 2008.

\section{Statement of Informed Consent}

Informed consent was obtained from all patients for being included in the study.

\section{REFERENCES}

1. Bhalerao J, Bowcock A. The genetics of psoriasis: a complex disorder of the skin and immune system. Hum Mol Genet. 1998;7:1537-45.

2. Trembath RC, Clough RL, Rosbotham JL, Jones AB, Camp RD, Frodsham A. Identification of a major susceptibility locus on chromosome $6 \mathrm{p}$ and evidence for further disease loci revealed by a two stage genome-wide search in psoriasis. Hum Mol Genet. 1997;6:813-20.

3. Tomfohrde J, Silverman A, Barnes R, Fernandez-Vina MA, Young M, Lory D. Gene for familial psoriasis susceptibility mapped to the distal end of human chromosome 17q. Science. 1994;264:1141-5.

4. Matthews D, Fry L, Powles A, Weber J, McCarthy M, Fisher E. Evidence that a locus for familial psoriasis maps to chromosome 4q. Nature Genet. 1996;14:231-3.

5. Capon F. Identification of ZNF313/RNF114 as a novel psoriasis susceptibility gene. Hum Mol Genet. 2008;17:1938-45.

6. Enlund F, Samuelsson L, Enerback C, Inerot A, Wahlstrom J, Yhr M. Psoriasis susceptibility locus in chromosome region 3q21 identified in patients from southwest Sweden. Eur J Hum Genet. 1999;7:783-90.

7. Lee YA, Ruschendorf F, Windemuth C, Schmitt-Egenolf M, Stadelmann A, Nurnberg G. Genomewide scan in German families reveals evidence for a novel psoriasis-susceptibility locus on chromosome 19p13. Am J Hum Genet. 2000;67:1020-4.

8. Veal CD, Clough RL, Barber RC, Mason S, Tillman D, Ferry B. Identification of a novel psoriasis susceptibility locus at $1 \mathrm{p}$ and evidence of epistasis between PSORS1 and candidate loci. J Med Genet. 2001;38:7-13.

9. Zhang XJ, He PP, Wang ZX, Zhang J, Li YB, Wang HY. Evidence for a major psoriasis susceptibility locus at 6p21 (PSORS1) and a novel candidate region at $4 \mathrm{q} 31$ by genome-wide scan in Chinese Hans. J Invest Dermatol. 2002;119:1361-6.

10. Liu Y, Helms C, Liao W, Zaba LC, Duan S, Gardner J. A genomewide association study of psoriasis and psoriatic arthritis identifies new disease loci. PLoS Genet. 2008;4:e1000041.

11. Nair RP. Genome-wide scan reveals association of psoriasis with IL-23 and NF-kappaB pathways. Nat Genet. 2009;41:199-204.

12. Zhang XJ. Psoriasis genome-wide association study identifies susceptibility variants within LCE gene cluster at 1q21. Nat Genet. 2009;41:205-10.

13. Tsunemi Y. Interleukin-12 p40 gene (IL12B) 3'- untranslated region polymorphism is associated with susceptibility to atopic dermatitis and psoriasis vulgaris. J Dermatol Sci. 2002;30:161-6.

14. Huffmeier U. Systematic linkage disequilibrium analysis of SLC12A8 at PSORS5 confirms a role in susceptibility to psoriasis vulgaris. J Invest Dermatol. 2005;125:906-12.

15. Hollox EJ. Psoriasis is associated with increased defensing genomic copy number. Nat Genet. 2008;40:23-5.

16. Feng BJ. Multiple loci within the major histocompatibility complex confer risk of psoriasis. PLoS Genet. 2009;5:e1000606.

17. Mischke D. Genes encoding structural proteins of epidermal cornification and S100 calcium-binding proteins form a gene complex ('epidermal differentiation complex') on human chromosome 1q21. J Invest Dermatol. 1996;106:989-92.

18. Bowcock AM, Krueger JG Getting under the skin. The immunogenetics of psoriasis. Nat Rev Immunol. 2005;5:699-711.

19. Begovich AB. The autoimmune disease-associated IL12B and IL23R polymorphisms in multiple sclerosis. Hum Immunol. 2007;68:934-7.

20. Duerr RH. A genome-wide association study identifies IL23R as an inflammatory bowel disease gene. Science. 2006;314:1461-3.

21. Van Beelen AJ. Stimulation of the intracellular bacterial sensor NOD2 programs dendritic cells to promote interleukin-17 production in human memory T cells. Immunity. 2007;27:660-9.

22. Fitzgerald O, Dougados M. Psoriatic arthritis: one or more diseases? Best Prac Res Clin Rheumatol. 2006;20:435-50.

23. Duffin KC, Chandran V, Gladman DD, Krueger GG, Elder JT, Rahman P. Genetics of psoriasis and psoriatic arthritis: Update and future direction. J Rheumatol. 2008;35:1449-53.

24. Gladman DD, Anhorn KA, Schachter RK, Mervart H. HLA antigens in psoriatic arthritis. J Rheumatol. 1986;13:586-92.

25. Holm SJ, Sánchez F, Carlén LM, Mallbris L, Ståhle M, O’Brien KP. HLA-Cw*0602 associates more strongly to psoriasis in the Swedish population than variants of the novel 6p21.3 gene PSORS1C3. Acta Derm Venereol. 2005;85:2-8.

26. Gonzalez S, Martinez-Borra J, Del Río JS, Santos-Juanes J, LopezVazquez A, Blanco-Gelaz M. The OTF3 gene polymorphism confers susceptibility to psoriasis independent of the association of HLA-Cw*0602. J Invest Dermatol. 2000;115:824-8.

27. Asumalahti K, Veal C, Laitinen T, Suomela S, Allen M, Elomaa O. Coding haplotype analysis supports $\mathrm{HCR}$ as the putative susceptibility gene for psoriasis at the MHC PSORS1 locus. Hum Mol Genet. 2002;11:589-97.

28. Holm SJ, Carlén LM, Mallbris L, Ståhle-Bäckdahl M, O’Brien KP. Polymorphisms in the SEEK1 and SPR1 genes on 6p21.3 associate with psoriasis in the Swedish population. Exp Dermatol. 2003;12:435-44.

29. Orrú S, Giuressi E, Carcassi C, Casula M, Contu L. Mapping of the major psoriasis-susceptibility locus (PSORS1) in a 70-Kb interval around the corneodesmosin gene (CDSN). Am J Hum Genet. 2005;76:164-71.

30. LiC, Wang G, Gao Y,Liu L, Gao T.TNF-alpha gene promoter-238G>A and $-308 \mathrm{G}>\mathrm{A}$ polymorphisms alter risk of psoriasis vulgaris: A metaanalysis. J Invest Dermatol. 2007;127:1886-92.

31. Reich K, Hüffmeier U, König IR, Lascorz J, Lohmann J, Wendler J. TNF polymorphisms in psoriasis: Association of psoriatic arthritis 
with the promoter polymorphism TNF*-857 independent of the PSORS1 risk allele. Arthritis Rheum. 2007;6:2056-64.

32. Nair RP, Stuart PE, Nistor I, Hiremagalore R, Chia NV, Jenisch S. Sequence and haplotype analysis supports HLA-C as the psoriasis susceptibility 1 gene. Am J Hum Genet. 2006;78:827-51.

33. Rahman P, Siannis F, Butt C, Farewell V, Peddle L, Pellett F. TNF alpha polymorphisms and risk of psoriatic arthritis. Ann Rheum Dis. 2006;65:919-23.

34. Rahman P, Roslin NM, Pellett F, Paterson A, Beyene J, Lemire M., High-resolution association mapping in the $\mathrm{MHC}$ region identifies multiple independent loci for psoriatic arthritis. Arthritis Rheum. 2009;60:S536.

35. Rahman P, Inman RD, Maksymowych WP, Reeve JP, Peddle L, Gladman DD. Association of interleukin 23 receptor variants with psoriatic arthritis. J Rheumatol. 2009;36:137-40.

36. Nelson GW, Martin MP, Gladman D, Wade J, Trowsdale J, Carrington M. Cutting edge: heterozygote advantage in autoimmune disease: Hierarchy of protection/susceptibility conferred by HLA and killer Ig-like receptor combinations in psoriatic arthritis. J Immunol. 2004;173:4273-6.

37. Setty AR, Curhan G, Choi HK. Obesity, waist circumference, weight change, and the risk of psoriasis in women: Nurses' Health Study II. Arch Intern Med. 2007;167:1670-5.

38. Tarantino G, Saldalamacchia G, Conca P, Arena A. Non-alcoholic fatty liver disease: further expression of the metabolic syndrome. J Gastroenterol Hepatol. 2007;22:293-303.

39. Ong JP, Pitts A, Younossi ZM. Increased overall mortality and liver related mortality in non-alcoholic fatty liver disease. J Hepatol. 2008;49:608-12.

40. Gisondi P, Targher G, Zoppini G, Girolomoni G. Non-alchohlic fatty liver disease in patients with chronic plaques disease. J Hepatol. 2009;51:758-64.

41. Al-Nozha MM, Al-Mazrou YY, Al-Maatouq MA, Arafah MR, Khalil MZ, Khan NZ, et al. Obesity in Saudi Arabia. Saudi Med J. 2005;26:824-9.

42. Alhyas L, McKay A, Balasanthiran A, Majeed A. Prevalences of overweight, obesity, hyperglycaemia, hypertension and dyslipidaemia in the Gulf: systematic review. J R Soc Med Sh Rep. 2011;2:55.

43. Libby P. Role of inflammation in atherosclerosis associated with rheumatoid arthritis. Am J Med. 2008;121(10, Suppl 1):S21-31.

44. Nickoloff BJ, Xin H, Nestle FO, Qin JZ. The cytokine and chemokine network in psoriasis. Clin Dermatol. 2007;25:568-73.

45. Croce $\mathrm{K}$, Libby P. Intertwining of thrombosis and inflammation in atherosclerosis. Curr Opin Hematol. 2007;14:55-61.

46. Spa"h F. Inflammation in atherosclerosis and psoriasis: common pathogenic mechanisms and the potential for an integrated treatment approach. Br J Dermatol. 2008;159(Suppl 2):10-7.

47. Targher G, Day, CP, Bonora E. Risk of cardiovascular disease in patients with nonalcoholic fatty liver disease. New England J Med. 2010;363:1341-50.

48. Targher G, Marra F, Marchesini G. Increased risk of cardiovascular disease in non-alcoholic fatty liver disease: causal effect or epiphenomenon? Diabetologia. 2008;51:1947-53.

49. Akahoshi M, Amasaki Y, Soda M. Correlation between fatty liver and coronary risk factors: A population study of elderly men and women in Nagasaki, Japan. Hypertens Res. 2001;24:337-43.

50. Rotman Y, Koh C, Zmuda JM, Kleiner DE, Liang TJ. The association of genetic variability in patatin-like phospholipase domain-containing protein 3 (PNPLA3) with histological severity of nonalcoholic fatty liver disease. Hepatol. 2010;52:894-903.

51. Romeo S, Kozlitina J, Xing C. Genetic variation in PNPLA3 confers susceptibility to nonalcoholic fatty liver disease. Nat Genet. 2008;40:1461-5.

52. Yuan X, Waterworth D, Perry JR. Population-based genome-wide association studies reveal six loci influencing plasma levels of liver enzymes. Am J Hum Genet. 2008;83:520-8.

53. Romeo S, Sentinelli F, Dash S. Morbid obesity exposes the association between PNPLA3 I148M (rs738409) and indices of hepatic injury in individuals of European descent. Int J Obes (Lond). 2010;34:190-4.

54. Kotronen A, Johansson LE, Johansson LM. A common variant in PNPLA3, which encodes adiponutrin, is associated with liver fat content in humans. Diabetologia. 2009;52:1056-60.

55. Anstee QM, Day CP. The genetics of NAFLD. Nat Rev Gastroenterol Hepatol. 2013;:645-55.

56. Anstee QM, Daly AK, Day CP. Genetics of alcoholic and nonalcoholic fatty liver disease. Semin Liver Dis. 2011;31:128-46.

57. He S, McPhaul C, Li JZ. A sequence variation (I148M) in PNPLA3 associated with nonalcoholic fatty liver disease disrupts triglyceride hydrolysis. J Biol Chem. 2010;285:6706-15.

58. Basantani MK, Sitnick MT, Cai L. Pnpla3/Adiponutrin deficiency in mice does not contribute to fatty liver disease or metabolic syndrome. J Lipid Res. 2011;52:318-29.

59. Chen W, Chang B, Li L, Chan L. Patatin-like phospholipase domaincontaining 3/adiponutrin deficiency in mice is not associated with fatty liver disease. Hepatol. 2010;52:1134-42.

60. Huang Y, He S, Li JZ. A feed-forward loop amplifies nutritional regulation of PNPLA3. Proc Natl Acad Sci USA. 2010;107:7892-7.

61. Goland S, Shimoni S, Zornitzki T, Knobler H, Azoulai O. Cardiac abnormalities as a new manifestation of nonalcoholic fatty liver disease: echocardiographic and tissue Doppler imaging assessment. J Clin Gastroenterol. 2006;40:949-55.

62. Yilmaz Y, Kurt R, Yonal O, Polat N, Celikel CA. Coronary flow reserve is impaired in patients with nonalcoholic fatty liver disease: association with liver fibrosis. Atherosclerosis. 2010;211:182-6.

63. Warner JP, Leek JP, Intody S, Markham AF, Bonthron DT. Human glucokinase regulatory protein $(\mathrm{GCKR})$ : cDNA and genomic cloning, complete primary structure, and chromosomal localization. Mamm Genome. 1995;6:532-6.

64. Køster B, Fenger M, Poulsen P, Vaag A, Bentzen J. Novel polymorphisms in the GCKR gene and their influence on glucose and insulin levels in a Danish twin population. Diabet Med. 2005;22:1677-82.

65. Willer CJ, Sanna S, Jackson AU, Scuteri A, Bonnycastle LL, Clarke R. Newly identified loci that influence lipid concentrations and risk of coronary artery disease. Nat Genet. 2008;40:161-9.

66. Okser S, Lehtimäki T, Elo LL, Mononen N, Peltonen N, Kähönen M. Genetic variants and their interactions in the prediction of increased pre-clinical carotid atherosclerosis: the cardiovascular risk in young Finns study. PLoS Genet. 2010;6: e1001146.

Copyright by lqbal Bukhari,, et al. This is an open-access article distributed under the terms of the Creative Commons Attribution License, which permits unrestricted use, distribution, and reproduction in any medium, provided the original author and source are credited.

Source of Support: Nil, Conflict of Interest: None declared. 